12) Nagata, S. and T. Yokoyama: Kagaku Kōgaku, 20, 272 (1965).

13) Nishikawa, M., Y. Okamoto, K. Hashimoto and S. Nagata: J. Chem. Eng. Japan, 9, 489 (1976).

14) Okamoto, Y., M. Nishikawa and K. Hashimoto: Int Chem. Eng., 21, 88 (1981).

15) Shinnar, R. and J. M. Church: Ind. Eng. Chem., 52, 253 (1960).

16) Sprow, F. B.: Chem. Eng. Sci., 22, 435 (1967).
17) Taylor, G. I.: Proc. Roy. Soc. London, 146A, 501 (1934), 151A, 421 (1935) and 164A, 476 (1938).

18) Van Heuven, J. W. and J. C. Hoevenaar: Proc. 4th Int. Symp. React. Eng., Bruxelles, 1969.

19) Vermulen, T., G. M. Williams and G. E. Langlois, Chem. Eng. Progr., 2, 85F (1955).

20) Yamaguchi, I., S. Tabuta and S. Nagata: Kagaku Kōgaku, 27, 576 (1963).

21) Yoshida, F. and T. Yamada: J. Ferment. Tech., 49, 235 (1971).

\title{
A THEORETICAL AND EXPERIMENTAL STUDY ON THE OSCILLATION OF A HANGING DROP
}

\author{
TAKAO TSUKADA, MASAKAZU SATO, NOBUYUKI IMAISHI, \\ MITSUNORI HOZAWA AND KATSUHIKO FUJINAWA \\ Chemical Research Institute of Non-Aqueous Solutions, \\ Tohoku University, Sendai 980
}

Key Words: Oscillation, Hanging Drop, Flow Pattern, Numerical Simulation, Drop Shape, Finite Element Method, Lagrangian Eulerian Description

\begin{abstract}
The damping oscillation of a hanging drop on a nozzle in the air was studied theoretically and experimentally. In the experiments, an elongated drop on a brass nozzle by electrostatic force was made to oscillate by removing the electric field instantaneously and the subsequent drop motion was recorded by a high-speed camera. In the theoretical study, the time-dependent drop shape and flow inside the drop were simulated numerically by use of the finite element method. It was found that the experimental results of oscillatory behavior for a hanging drop of aqueous glycerin solution were in good agreement with the calculated ones and that the frequency of oscillation was affected by drop volume, surface tension and nozzle size.
\end{abstract}

\section{Introduction}

It is of practical interest to know the behavior of bubbles or drops moving in a fluid. However, as the shape of the two-fluid interface usually changes with time, ${ }^{6,10,11,15)}$ a strict analysis is very difficult.

In this work, numerical simulations of the oscillations of a hanging drop on a nozzle in the air were carried out, as the first step in the analysis of the oscillatory behavior of moving drops or bubbles in other fluids.

Rayleigh $^{9)}$ is the pioneer for this problem. He attempted to analyze theoretically the small-amplitude oscillations of an inviscid drop. Lamb ${ }^{5\}}$ extended the work by considering the density effect of the surrounding fluid.

Miller and Scriven ${ }^{7}$ solved a similar problem, taking the viscosity of the two fluids into account.

\footnotetext{
Received May 31, 1986. Correspondence concerning this article should be addressed to T. Tsukada. M. Sato is now with Shin-Etsu Handotai Co., Ltd., Nishishirakawagun 961 .

Recently, Prosperetti ${ }^{8)}$ calculated the initial value problem for the linear free oscillation of a bubble or a drop, and Tsamopoulous et al. ${ }^{12)}$ analyzed theoretically the moderate-amplitude nonlinear oscillations of inviscid drops and bubbles using a PoincaréLindstedt expansion technique.

However, there have been few studies ${ }^{13}$ in which the full Navier-Stokes equations for the oscillations of moving bubbles or drops are solved.

Hughes et al. ${ }^{3)}$ and Kheshgi et al. ${ }^{4)}$ reported that it was efficient to use the finite element method including the Lagrangian-Eulerian description for the calculation of the transient flow problem with free surface.

The first aim of this work is to analyze theoretically the oscillations of a hanging drop by use of the finite element method. ${ }^{3,4,13)}$ The second aim is to confirm experimentally the propriety of the calculation method and to identify the factors that affect the oscillations. 


\section{Theory}

Figure 1 shows the coordinate systems for the numerical analysis of the oscillations of a hanging drop in the air. The cylindrical coordinates are used for the calculation of flow inside the drop and the spherical ones are used for that of the drop profile. The drop shape is assumed to change with time from the initial profile shown by the dotted line in Fig. 1 to the equilibrium profile of the solid line.

The unsteady-state Navier-Stokes equation and the continuity equation for the drop phase are described by Eqs. (1) and (2) in dimensionless form, respectively.

$$
\begin{gathered}
\partial \boldsymbol{v} / \partial t=-\boldsymbol{v} \cdot \nabla \boldsymbol{v}+\nabla \cdot \boldsymbol{T}+G \boldsymbol{e}_{z} \\
\nabla \cdot \boldsymbol{v}=0
\end{gathered}
$$

where $T$ is a stress tensor $\left(T=-p \boldsymbol{I}+\left(\nabla \boldsymbol{v}+\nabla \boldsymbol{v}^{T}\right)\right)$ and $G$ is a gravitational parameter defined as $R_{a}^{\prime 3} g / v^{2}$.

The dimensionless boundary conditions are expressed by Eq. (3).

at the nozzle tip: $\boldsymbol{n} \cdot \boldsymbol{v}=0, \boldsymbol{t} \cdot \boldsymbol{v}=\mathbf{0}$

at the center line: $\boldsymbol{n} \cdot \boldsymbol{v}=0, \quad \boldsymbol{\tau}: \boldsymbol{n t}=\mathbf{0}$

at the drop surface:

$$
\begin{aligned}
& \left.\boldsymbol{n} \cdot \boldsymbol{v}=f(\partial f / \partial t) / \sqrt{f^{2}+(\partial \bar{f} / \partial \bar{\theta}}\right)^{2} \\
& \boldsymbol{\tau}: \boldsymbol{n} \boldsymbol{t}=0
\end{aligned}
$$

Ca $T: \boldsymbol{n n}=2 H$

Equation (3-5) means that fluid elements do not cross the drop surface but move with it. This is called the kinematic condition.

The initial conditions are given as follows.

$$
\boldsymbol{v}=0, f=f_{0}(\theta)
$$

To reduce the number of unknown variables, the penalty formulation ${ }^{3,4)}$ of the imcompressible constraint was introduced. Pressure is approximated by Eq. (5) using the penalty parameter $\lambda$ and is substituted into Eq. (1).

$$
p=-\lambda(\nabla \cdot \boldsymbol{v})
$$

where a $\lambda$ value of $10^{8}$ is chosen.

To solve the above equations, the Galerkin finite element $\operatorname{method}^{3,4,13)}$ was used. Each finite element consists of a four-node quadrilateral, and the dimensionless velocity vector $v$ is approximated as follows.

$$
v=\sum \phi_{i} v_{i}
$$

where $\phi_{i}$ is a bilinear trial function.

Substituting Eq. (6) into Eq. (1), the Galerkin formulation of Eq. (1) is given by Eq. (7).

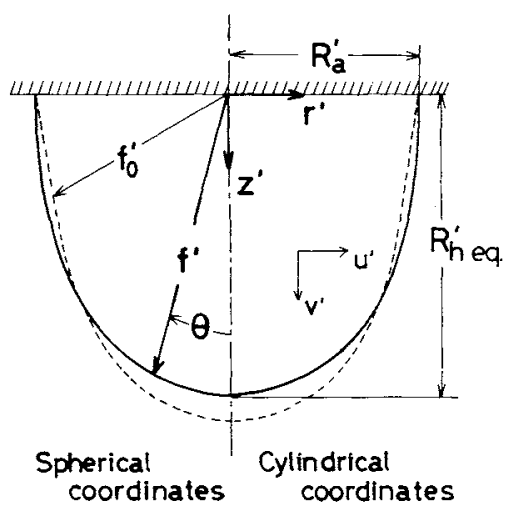

Fig. 1. Coordinate systems.

$$
\begin{gathered}
\int_{V}\left\{\phi_{i} \partial \boldsymbol{v} / \partial t+\phi_{i} \boldsymbol{v} \cdot \boldsymbol{\nabla} \boldsymbol{v}+\nabla \phi_{i} \cdot \boldsymbol{T}-\phi_{i} G \boldsymbol{e}_{z}\right\} d V \\
-\int_{S} \phi_{i}\{\boldsymbol{T}: \boldsymbol{n n n}+\boldsymbol{T}: \boldsymbol{n t t}\} d S=0
\end{gathered}
$$

where $\boldsymbol{T}$ is expressed using $\lambda$ as $\boldsymbol{T}=\lambda(\boldsymbol{V} \cdot \boldsymbol{v}) \boldsymbol{I}+$ $\left(\nabla v+\nabla v^{T}\right)$. In applying Eq. (3-7) to the boundary integration term of Eq. (7), mean curvature $2 H$ is expressed by Eq. (8) using the spherical coordinates.

$$
\begin{aligned}
2 H= & \left\{f\left(\partial^{2} f / \partial \theta^{2}\right)-(\partial f / \partial \theta)^{2}\right\} /\left\{f^{2}+(\partial f / \partial \theta)^{2}\right\}^{3 / 2} \\
& +\left\{(\partial f / \partial \theta) \cos \theta-2 f^{\prime}\right\} / f /\left\{f^{2}+(\partial f / \partial \theta)^{2}\right\}^{1 / 2}
\end{aligned}
$$

where $(\partial f / \partial \theta)$ and $\left(\partial^{2} f / \partial \theta^{2}\right)$ are obtained by approximating the drop shape by a spline function.

As the drop surface changes with time, the finite elements ought to move together. One of the methods of describing the moving boundary is the Lagrangian method, but it is inconvenient for a system with large deformation because each element deforms with time arbitrarily. Therefore, the Lagrangian-Eulerian description, ${ }^{3,4)}$ which allows the elements to move along the appropriate straight line, that is, in the radial direction in spherical coordinates as shown in Fig. 2, is used in this work. To calculate the radial component $\bar{v}_{f}$ of the moving velocity of the elements, the relation between $\bar{v}_{f}$ and $v\left(=u_{f} \boldsymbol{e}_{f}+u_{\theta} \boldsymbol{e}_{\theta}\right)$ is given as follows.

$$
\bar{v}_{f}=\alpha\left\{u_{f}-(1 / f)(\partial f / \partial \theta) u_{0}\right\}
$$

where $\alpha$ is the Lagrangian-Eulerian parameter. $\alpha$ is equal to 1 if the element is made to move and zero if not. By applying the Galerkin finite element method to Eq. (9), the moving velocity of each element can be calculated.

Accordingly, the Galerkin formulation of the dimensionless Navier-Stokes equation is expressed by Eq. (10), substituting the moving velocity of the element into the inertia term of Eq. (7).

$$
\begin{gathered}
\int_{V}\left\{\phi_{i} \partial \boldsymbol{v} / \partial t+\phi_{i}(\boldsymbol{v}-\overline{\boldsymbol{v}}) \cdot \nabla \boldsymbol{v}+\nabla \phi_{i} \cdot \boldsymbol{T}-\phi_{i} G \boldsymbol{e}_{z}\right\} d V \\
\quad-\int_{S} \phi_{i}(\boldsymbol{T}: \boldsymbol{n n n}+\boldsymbol{T}: \boldsymbol{n t}) d S=0
\end{gathered}
$$




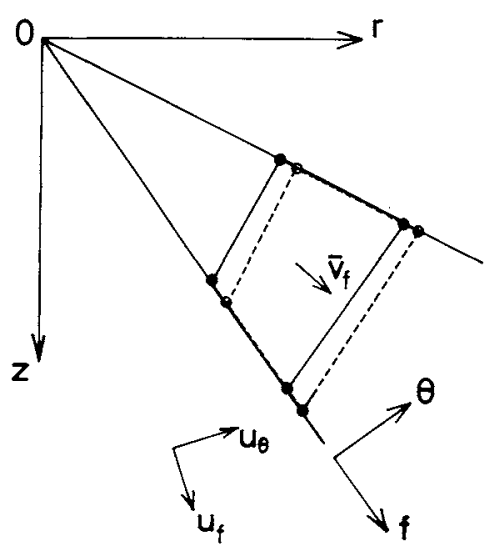

Fig. 2. Moving finite element.

where

$$
\begin{gathered}
v=\left(u_{f} \sin \theta+u_{\theta} \cos \theta\right) e_{r}+\left(u_{f} \cos \theta-u_{\theta} \sin \theta\right) \boldsymbol{e}_{z} \\
\overline{\boldsymbol{v}}=\bar{v}_{f} \sin \theta \boldsymbol{e}_{\boldsymbol{r}}+\bar{v}_{f} \cos \theta \boldsymbol{e}_{z}
\end{gathered}
$$

The flow field is determined by solving Eq. (10) under the initial and the boundary conditions. For the inertia term of Eq. (10), the upwind finite element method developed by Hughes et al. ${ }^{3)}$ was used to preclude a spurious oscillation of the solution. To solve a set of algebraic equations generated from Eq. (10), the frontal method by Hood $^{2)}$ was used.

\section{Experimental Apparatus and Procedure}

To obtain an initial drop shape which is slightly different from the equilibrium shape in the gravitational field, drop formation in an electrostatic field ${ }^{14)}$ was utilized here.

Figure 3 shows the experimental apparatus, which is almost the same as that of the previous work. ${ }^{14)} \mathrm{A}$ single drop of arbitrary volume was hung on the brass nozzle which was set in the center of the upper electrode (brass plate of $150 \times 150 \mathrm{~mm}$ ), and D.C. high voltage $(5-15 \mathrm{kV})$ was applied. After the drop was elongated appropriately by the electrostatic force, the voltage was set to zero instantaneously and the oscillation of the drop was photographed continuously both by streak photography using a camera (Nikon F2 with $200 \mathrm{~mm}$ lens) with a stroboscope and by use of a $16 \mathrm{~mm}$ high-speed camera. The framing rate in the two techniques was 100 and 400 frames per second, respectively. The drop volume was calculated from the numerical integration of the initial profile. The nozzle radius was $1.5,2$ and $2.5 \mathrm{~mm}$, and the distance between the electrodes was $3.5 \mathrm{~cm}$. Distilled water and aqueous glycerin solutions were used as the drop phase. Their physical properties are listed in Table 1.

The whole experimental system was set in an air bath at $298 \mathrm{~K}$.

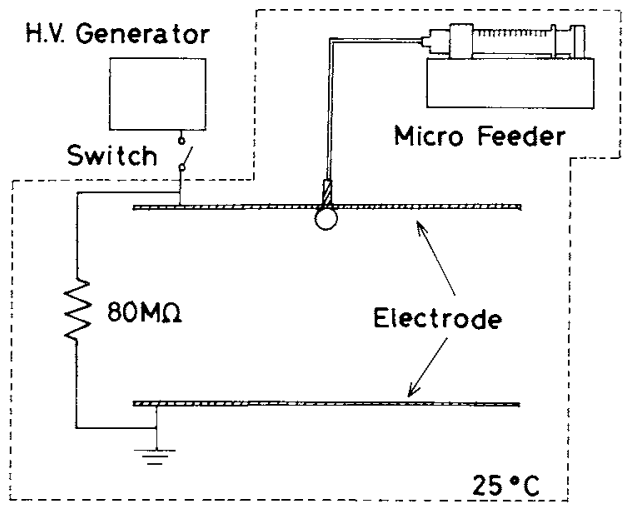

Fig. 3. Experimental apparatus.

Table 1. Physical properties of liquid employed (at $298 \mathrm{~K}$ )

\begin{tabular}{lrlc}
\hline \multicolumn{1}{c}{ Liquid } & $\rho\left[\mathrm{kg} / \mathrm{m}^{3}\right]$ & $\mu[\mathrm{Pa} \cdot \mathrm{s}]$ & $\gamma[\mathrm{mN} / \mathrm{m}]$ \\
\hline Water & 997 & 0.00089 & 72.0 \\
Aqueous glycerin soln. 1 & 1150 & 0.0095 & 66.8 \\
Aqueous glycerin soln. 2 & 1170 & 0.015 & 65.5 \\
Aqueous glycerin soln. 3 & 1180 & 0.025 & 64.6 \\
Aqueous glycerin soln. 4 & 1190 & 0.028 & 64.8 \\
\hline
\end{tabular}

\section{Results and Discussion}

\subsection{Comparison of numerical and experimental re- sults}

Figure 4 shows the comparison of the numerical and experimental results for the oscillation of a hanging drop of aqueous glycerin solution, where $R_{h e q}-R_{h}$ is the difference between the dimensionless drop height in the equilibrium state and that during oscillation on the $z$ axis. The dotted line indicates the fitting curve of the experimental results by use of the following equation for damped oscillation.

$R_{\text {heq. }}-R_{h}=\left(R_{\text {heq. }}-R_{h 0}\right) \exp \left(-\beta t^{\prime}\right) \cos \left(2 \pi \omega^{*} t^{\prime}\right)$

where $\beta$ is damping coefficient, $\omega^{*}$ is frequency and $R_{h 0}$ is the dimensionless drop height at $t^{\prime}=0$.

Apparently, the calculated result is in good agreement with the experimental one where the frequency is $18.4 \mathrm{~Hz}$ in the experiment and $18.7 \mathrm{~Hz}$ in the calculation respectively.

Figure 5 shows the calculated flow pattern inside the drop and the element arrangement at $t^{\prime}=0.0081 \mathrm{~s}$ and $t^{\prime}=0.035 \mathrm{~s}$ where the conditions are the same as in Fig. 4. The drop shape shown by the dotted line is the equilibrium shape. It is seen that the flow direction inside the drop changes periodically and the nodal points move along the radial direction in spherical coordinates according to the oscillation.

Figure 6 shows the experimental and calculated results under the same conditions as those in Fig. 4 except for the drop volume (about $80 \%$ smaller than that in Fig. 4). In this case, the experimental frequency is $25.1 \mathrm{~Hz}$ and the calculated one is $25.3 \mathrm{~Hz}$. 


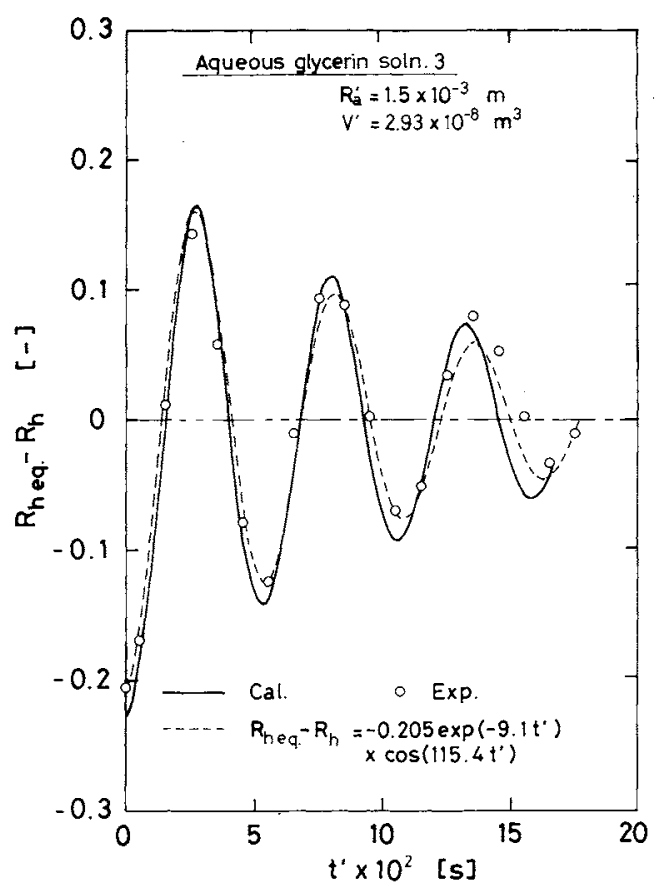

Fig. 4. Comparison of numerical and experimental results for oscillation of a hanging drop.
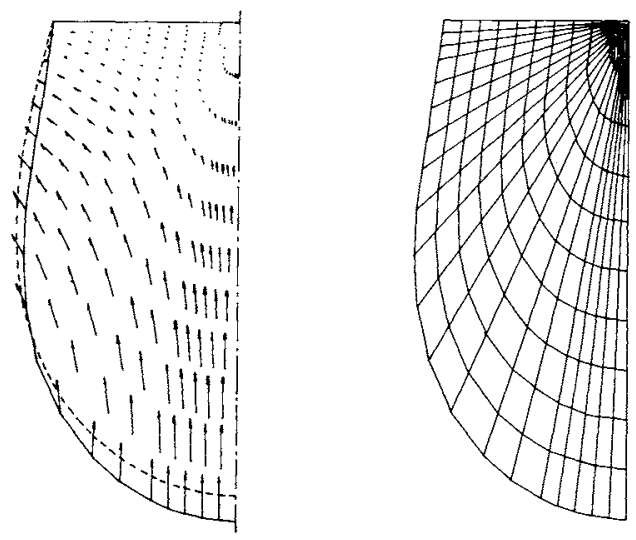

(a) $t^{\prime}=0.0081 \mathrm{~s}$
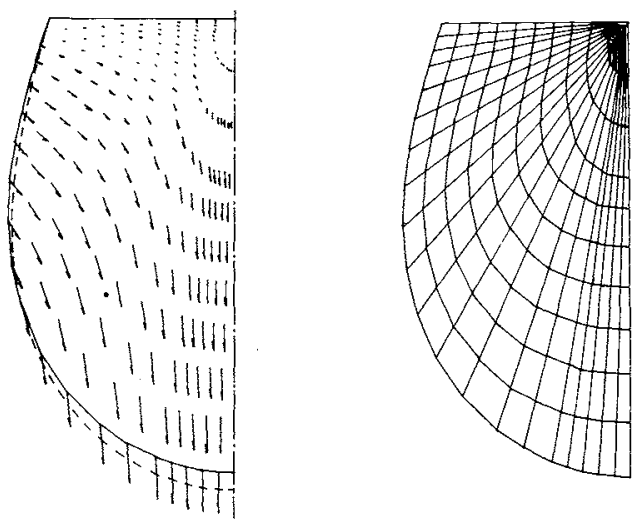

(b) $t^{\prime}=0.035 \mathrm{~s}$

Fig. 5. Calculated flow pattern inside the drop and the element arrangement.

Apparently, the frequency becomes larger as $V^{\prime}$ decreases, in comparison with that in Fig. 4.

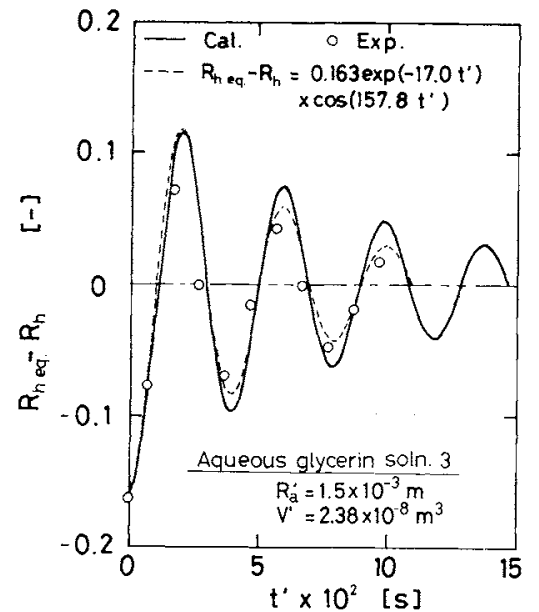

Fig. 6. Comparison of numerical and experimental results for oscillation of a hanging drop.

\subsection{Factors affecting the frequency}

1) Drop volume Figure 7 shows the experimental relations between the frequency $\omega^{*}$ and the drop volume $V^{\prime}$ for various concentrations of aqueous glycerin solutions where the nozzle radius is $2.5 \mathrm{~mm}$. Apparently, the frequency decreases as $V^{\prime}$ increases.

For Glycerin 4, the calculated results are also plotted in the region of small drop volume. Calculations of large $V^{\prime}$ values were not carried out because they require much computation time. Apparently, the experimental and the calculated results lie on one line, and the frequency is proportional to $V^{\prime-1.38}$

2) Viscosity From the comparison of the results for three kinds of aqueous glycerin solutions in Fig. 7, it is apparent that the effect of viscosity on the frequency is insignificant, although the damping coefficient increases as viscosity increases (for example, $\beta=2.77$ for Glycerin 1 and $\beta=8.33$ for Glycerin 4 , where $V^{\prime} \fallingdotseq 7.2 \times 10^{-8} \mathrm{~m}^{3}$ ).

Figure 8 shows the calculated results of the effect of viscosity on the oscillation of a hanging drop with the appropriate parameters, where $\rho$ and $\gamma$ are those for $75 \%$ glycerin solution. The frequency becomes slightly larger as viscosity decreases.

3). Surface tension Figure 9 shows the calculated results as to the effect of surface tension on the oscillatory behavior for the same conditions as those in Fig. 8. The frequency increases as surface tension increases. However, the effect of surface tension on $\omega^{*}$ is not represented by a simple power law such as $\omega^{*} \propto \gamma^{n}$.

4) Nozzle radius Figure $\mathbf{1 0}$ shows the effect of the nozzle radius on the experimental relations between $\omega^{*}$ and $V^{\prime}$. For the same drop volume, the frequency increases with nozzle radius. This result would be caused by the effect of the nozzle. It is also seen that the nozzle radius affects the slope of the line of $\omega^{*}$ vs. 


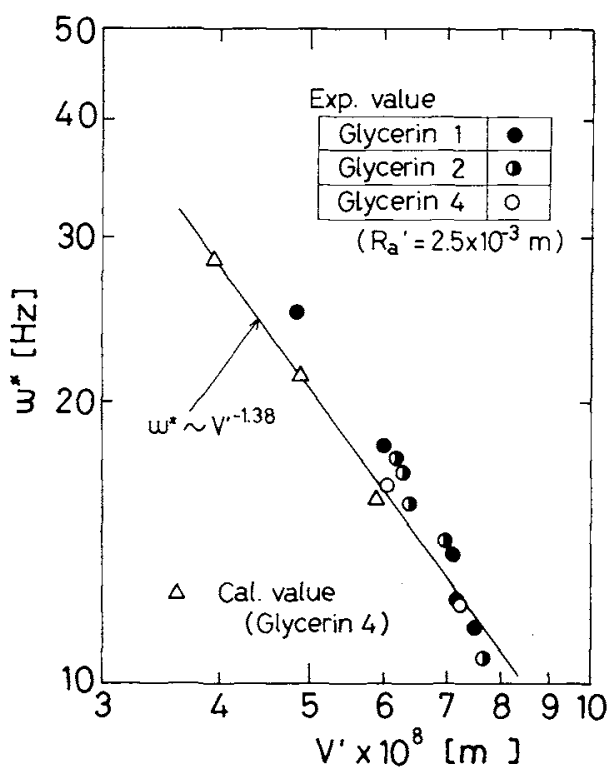

Fig. 7. Effect of drop volume on frequency.

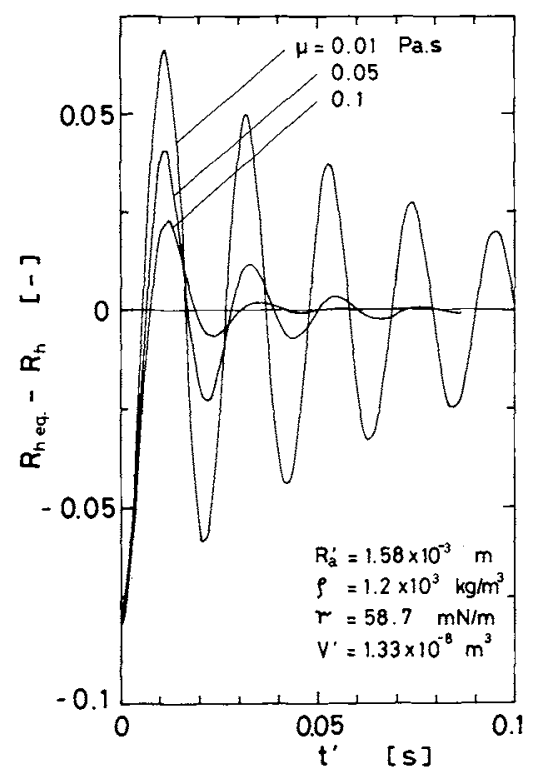

Fig. 8. Calculated results for effect of viscosity.

$V^{\prime}$ relation. The gradient of the solid line in Fig. 10 becomes smaller as $R_{a}^{\prime}$ decreases, that is, -1.68 , $-1.35,-1.18$ for $R_{a}^{\prime}=2.5,2.0$ and $1.5 \mathrm{~mm}$ respectively.

Lamb's natural frequency ${ }^{5}$ is expressed as follows for the fundamental mode of a spherical free drop.

$$
\omega^{*}=\left\{16 \gamma /\left(\pi^{2} d_{e}^{3} \rho\right)\right\}^{1 / 2}
$$

where $d_{e}$ is volume equivalent diameter, namely, $d_{e}^{3}$ corresponds to drop volume, and $\rho$ is density of the drop. The experimental and the calculated effects of $\gamma$ and $V^{\prime}$ on $\omega^{*}$ agree with Eq. (12) qualitatively, but the exponents for each factor are different from those in Eq. (12). The discrepancy seems to be due to the effect of the nozzle.

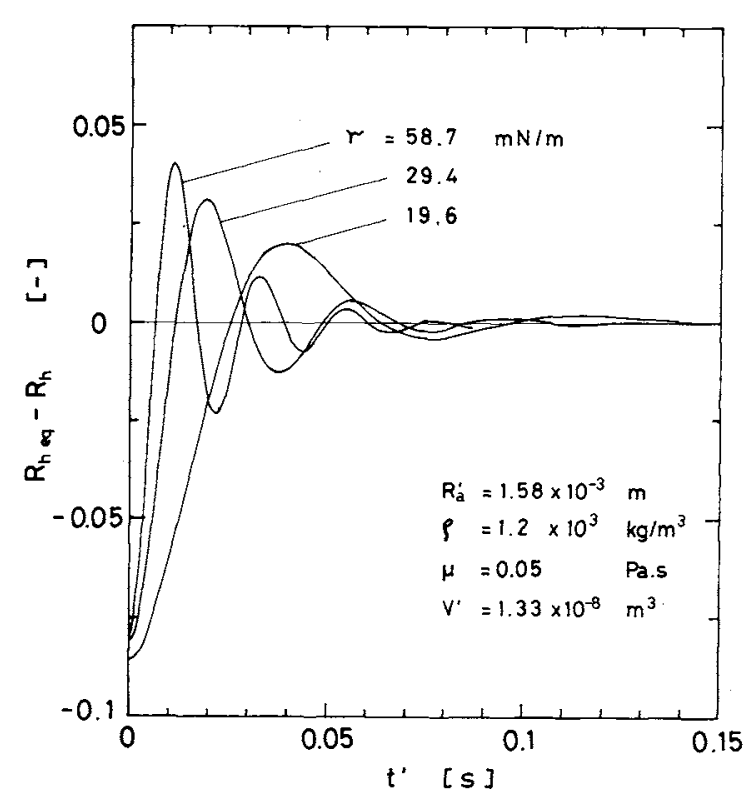

Fig. 9. Calculated effect of surface tension.

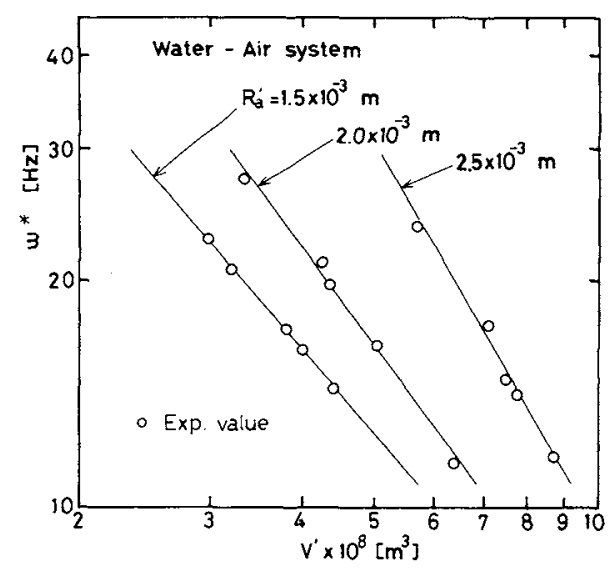

Fig. 10. Experimental effect of nozzle radius on frequency.

\section{Conclusion}

The oscillations of a hanging drop on a nozzle were studied experimentally and theoretically and the following conclusions were obtained.

The calculation method proposed here can predict the oscillatory behavior of a hanging drop, particularly the frequency.

The factors affecting the frequency are drop volume, surface tension and nozzle radius. The effect of viscosity on the frequency is insignificant.

\section{Acknowledgment}

This work was partially supported by Grant-in-Aid for Scientific Research 59550640 from the Ministry of Education, Science and Culture of Japan. The numerical calculations were carried out by use of ACOS 1000 of the Computer Center of Tohoku Univ.

\section{Nomenclature}

$\mathrm{Ca} \quad=$ Capillary number $\left(=\rho v^{2} / R_{u}^{\prime} \gamma\right)$

$\begin{array}{lll}d_{e} & =\text { volume equivalent diameter } & \text { [m] }\end{array}$ 


\begin{tabular}{|c|c|c|}
\hline$e_{z}$ & $=$ axial unit vector & {$[-]$} \\
\hline$f^{\prime \prime}$ & $=$ radial distance in spherical coordinates & {$[\mathrm{m}]$} \\
\hline$f_{0}^{\prime}$ & $=$ initial drop shape & [m] \\
\hline$f, f_{0}^{\prime}$ & $=f^{\prime} / R_{a}^{\prime}, f_{0}^{\prime} / R_{a}^{\prime}$ & {$[-]$} \\
\hline$G$ & $=$ gravitational parameter $\left(=R_{a}^{\prime 3} g / \nu^{2}\right)$ & {$[-]$} \\
\hline$g$ & $=$ gravitational acceleration & {$\left[\mathrm{m} / \mathrm{s}^{2}\right]$} \\
\hline$H^{\prime}$ & $=$ mean curveture & {$[1 / \mathrm{m}]$} \\
\hline$H$ & $=H^{\prime} R_{a}^{\prime}$ & {$[-]$} \\
\hline$I$ & $=$ unit tensor & {$[-]$} \\
\hline$n$ & $=$ unit vector normal to boundary surface & {$[-]$} \\
\hline$p^{\prime}$ & $=$ pressure & {$[\mathrm{Pa}]$} \\
\hline$p$ & $=R_{a}^{\prime 2}\left(p^{\prime}-p_{G}^{\prime}\right) / \rho v^{2}$ & {$[-]$} \\
\hline$p_{G}^{\prime}$ & $=$ uniform pressure in the air & [Pa] \\
\hline$R_{a}^{\prime}$ & $=$ nozzle radius & [m] \\
\hline$R_{h}^{\prime \prime}$ & $=$ drop height at $r^{\prime}=0$ & {$[\mathrm{~m}]$} \\
\hline$R_{h 0}^{\prime}$ & $=$ initial drop height at $r^{\prime}=0$ & [m] \\
\hline$R_{\text {heq }}^{\prime}$ & $\begin{array}{l}=\text { drop height in the equilibrium state at } \\
r^{\prime}=0\end{array}$ & {$[\mathrm{~m}]$} \\
\hline$R_{h}, R_{h 0}, R_{\text {heq }}$ & $=R_{h}^{\prime} / R_{a}^{\prime}, R_{h 0}^{\prime} / R_{a}^{\prime}, R_{h e q}^{\prime} / R_{a}^{\prime}$ & {$[\mathrm{m}]$} \\
\hline$r^{\prime}$ & $=$ radial distance in cylindrical coordinates & {$[\mathrm{m}]$} \\
\hline$r$ & $=r^{\prime} / R_{a}^{\prime}$ & {$[-]$} \\
\hline$T^{\prime}$ & $=$ stress tensor & {$[\mathrm{Pa}]$} \\
\hline$T$ & $=R_{a}^{\prime 2} T^{\prime} / \rho v^{2}$ & {$[-]$} \\
\hline$t^{\prime}$ & $=$ time & [s] \\
\hline$t$ & $=t^{\prime} v / R_{a}^{\prime 2}$ & {$[-]$} \\
\hline$t$ & $=$ tangential unit vector at boundary surface & {$[-]$} \\
\hline$u^{\prime}$ & $\begin{aligned}= & \text { radial component of velocity vector in } \\
& \text { cylindrical coordinates }\end{aligned}$ & {$[\mathrm{m} / \mathrm{s}]$} \\
\hline$u_{f}^{\prime}$ & $\begin{aligned}= & \text { radial component of velocity vector in spheri } \\
& \text { coordinates }\end{aligned}$ & $\begin{array}{l}\mathrm{rical} \\
{[\mathrm{m} / \mathrm{s}]}\end{array}$ \\
\hline$u_{\theta}^{\prime}$ & $\begin{array}{l}=\text { polar angle component of velocity vector } \\
\text { in spherical coordinates }\end{array}$ & {$[\mathrm{m} / \mathrm{s}]$} \\
\hline$u, u_{f}, u_{\theta}$ & $=u^{\prime} R_{a}^{\prime} / v, u_{f}^{\prime} R_{a}^{\prime} / v, u_{\theta}^{\prime} R_{a}^{\prime} / v$ & {$[-]$} \\
\hline & $=$ drop volume & {$\left[\mathrm{m}^{3}\right]$} \\
\hline$v^{\prime}$ & $\begin{aligned}= & \text { axial component of velocity vector in } \\
& \text { cylindrical coordinates }\end{aligned}$ & {$[\mathrm{m} / \mathrm{s}]$} \\
\hline$v^{\prime}$ & $=$ velocity vector & {$[\mathrm{m} / \mathrm{s}]$} \\
\hline $\bar{v}_{f}^{\prime}$ & $\begin{aligned}= & \text { radial component of moving velocity vector } \\
& \text { of element in spherical coordinates }\end{aligned}$ & {$[\mathrm{m} / \mathrm{s}]$} \\
\hline $\bar{v}^{\prime}$ & $=$ moving velocity vector of element & {$[\mathrm{m} / \mathrm{s}]$} \\
\hline$v, v, \bar{v}_{f}, \overline{\boldsymbol{v}}$ & $=v^{\prime} R_{a}^{\prime} / v, v^{\prime} R_{a}^{\prime} / v, \bar{v}_{f}^{\prime} R_{a}^{\prime} / v, \bar{v}^{\prime} R_{a}^{\prime} / v$ & {$[-]$} \\
\hline$z^{\prime}$ & $=$ axial distance in cylindrical coordinates & [m] \\
\hline
\end{tabular}

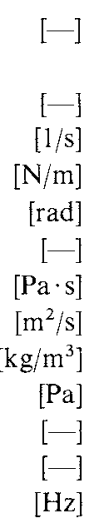

\section{Literature Cited}

1) Foote, G. B.: J. Comput. Phys., 11, 507 (1973).

2) Hood, P.: Int. J. Num. Meth. Eng., 10, 379 (1976).

3) Hughes, T. J. R., W. K. Liu and T. K. Zimmermann: "Proc. U.S.-Japan Conf. on Interdisciplinary Finite Element Analysis," (1978).

4) Kheshgi, H. S. and L. E. Scriven: "Finite Element in Fluids," Vol. 5, Wiley, London (1984).

5) Lamb, H.: "Hydrodynamics," 6th ed. Cambridge Univ. Press, London (1932).

6) Loshak, J. and C. H. Byers: Chem. Eng. Sci, 28, 149 (1973).

7) Miller, C. and L. E. Scriven: J. Fluid Mech., 32, 417 (1968).

8) Prosperetti, A.: J. Fluid Mech., 100, 333 (1980).

9) Rayleigh, J. W. S.: Proc. R. Soc. Lond., 29, 71 (1879).

10) Trinh, E., A. Zwern and T. G. Wang: J. Fluid Mech., 115, 453 (1982).

11) Trinh, E. and T. G. Wang: J. Fluid Mech., 122, 315 (1982).

12) Tsamopoulos. J. A. and R. A. Brown: J. Fluid Mech., 127, 519 (1983).

13) Tsukada, T., M. Hozawa, N. Imaishi and K. Fujinawa: $J$. Chem. Eng. Japan, 17, 246 (1984).

14) Tsukada, T., M. Sato, N. Imaishi, M. Hozawa and K. Fujinawa: J. Chem. Eng. Japan, 19, 537 (1986).

15) Valentine, R. S., N. F. Sather and W. J. Heideger: Chem. Eng. Sci., 20, 719 (1965). 